\title{
A Formula for Human Average Whole-Body SAR under Diffuse Fields Exposure in the GHz region
}

\author{
A Bamba*, W Joseph, G Vermeeren, A Thielens, E Tanghe, \\ and L Martens \\ Ghent University / iMinds - Department of Information Technology \\ Gaston Crommenlaan 8 box 201, B-9050 Ghent-Belgium \\ Fax: +3293314 899, Tel: +3293314908 \\ E-mail: aliou.bamba@intec.ugent.be
}

\begin{abstract}
.
A simple formula to determine the human average whole-body SAR $\left(\mathrm{SAR}_{w b}\right)$ under realistic propagation conditions is proposed in the $\mathrm{GHz}$ region, i.e., from $1.45 \mathrm{GHz}$ to $5.8 \mathrm{GHz}$. The methodology is based on simulations of ellipsoidal human body models. Only the exposure (incident power densities) and the human mass are needed to apply the formula. Diffuse scattered illumination is addressed for the first time and the possible presence of a Line-Of-Sight (LOS) component is addressed as well. As validation, the formula is applied to calculate the average whole-body $\mathrm{SAR}_{w b}$ in 3-D heterogeneous phantoms, i.e., the virtual family (34-year-old male, 26-year-old female, 11-year-old girl, and 6-year-old boy) and the results are compared with numerical ones - using the Finite-Difference Time-Domain (FDTD) method - at $3 \mathrm{GHz}$. For the LOS exposure, the average relative error varies from $28 \%$ to $12 \%$ (resp. $14 \%$ to $12 \%$ ) for the vertical polarization (resp. horizontal polarization), depending on the heteregeneous phantom. Regarding the diffuse illumination, relative errors of $39.40 \%,-11.70 \%, 10.70 \%$, and $10.60 \%$ are obtained for the 6 -year-old boy, 11 -year-old girl, 26-year-old female, and 34-year-old male, respectively. The proposed formula estimates well (especially for adults) the $\mathrm{SAR}_{w b}$ induced by diffuse illumination in realistic conditions.

In general, the correctness of the formula improves when the human mass increases. Keeping the uncertainties of the FDTD simulations in mind, the proposed formula might be important for the dosimetry community to assess rapidly and accurately the human absorption of electromagnetic radiation caused by diffuse fields in the $\mathrm{GHz}$ region.

Finally, we show the applicability of the proposed formula to personal dosimetry for epidemiological research.
\end{abstract}




\section{Introduction}

Due to the increasing deployment of wireless equipment, it is important to investigate the possible health effects of exposure to electromagnetic fields (EMF). The International Commission on Non-Ionizing Radiation Protection (ICNIRP 1998) defined limit values for the whole-body averaged specific energy absorption rate $\left(\mathrm{SAR}_{w b}\right)$ to prevent harmful effects of the EMF in the radio-frequency (RF) range. Several studies (Dimbylow 2002, Wang et al 2006, Kühn et al 2009) determined numerically the $\mathrm{SAR}_{w b}$ based on plane wave exposure. The $\mathrm{SAR}_{w b}$ depends on many parameters (posture, polarization of the incident plane wave, direction of the plane wave, human morphology, etc.) (ICNIRP 1998). A variability study of the $\mathrm{SAR}_{w b}$ for different morphological phantoms, for instance, can be found in Conil et al (2008). Vermeeren et al (2008b) investigated the $\mathrm{SAR}_{w b}$ in child phantoms in a realistic environment in the $\mathrm{MHz}$ range. The authors showed that the worst-case incident plane wave polarization depends on the ratio of the phantom size and the considered wavelength. This result is confirmed in Hirata et al (2009) and Uusitupa et al (2010) wherein it is stated that the polarization of the incident electric field may have an effect of several $\mathrm{dB}$ on the $\mathrm{SAR}_{w b}$. The vertical polarization based $\mathrm{SAR}_{w b}$ is higher up to the resonant frequency (order of 60-120 MHz), whereas the horizontal polarization result becomes higher in the $\mathrm{GHz}$ region (Uusitupa et al 2010). Obviously, the $\mathrm{SAR}_{w b}$ due to a single plane wave illumination depends on its direction of arrival: this is confirmed in Conil et al (2011) where it was shown that the $\mathrm{SAR}_{w b}$ varies periodically with the azimuth. This periodic behavior will occur for any other human phantom at the $\mathrm{GHz}$ frequencies since the physically illuminated body surface varies with the incident plane-wave azimuth.

Despite the large number of the parameters involved in the $\mathrm{SAR}_{w b}$ and their complexities, Hirata et al (2010) proposed a formula on the basis of the analogy of the human body and a half dipole antenna to determine the $\mathrm{SAR}_{w b}$ for plane-wave exposure at the resonance frequency. The authors highlighted the impact of the tissues inhomogeneity on the estimated $\mathrm{SAR}_{w b}$ at the resonance frequency. The derived formula accounts for the proportion of fat in the human model as well. All those previous works investigated the human whole-body $\mathrm{SAR}_{w b}$ due to a deterministic plane wave illumination.

Recently, Bamba et al (2012) developed a new measurement-based method to determine experimentally the $\mathrm{SAR}_{w b}$ of humans in a realistic indoor environment. The novelty of this method is that it accounts for the diffuse multipath components (DMC). The DMC is the part of the propagation data that can not be characterized with discrete plane waves (Richter et al 2003, Richter 2005, Poutanen et al 2011). Rather, the DMC is characterized by a continuous power density as function of azimuthal angle, timedelay, etc. Therefore, the diffuse fields can not be recorded separately by a personal exposure meter, by a network analyzer, nor by a spectrum analyzer, which record the total incident field.

The DMC occurs usually in realistic indoor environments. It has been shown that the 
DMC power density may contribute significantly - up to $95 \%$ - to the total power density in an indoor environment (Poutanen et al 2011). Furthermore, Bamba et al (2012) showed that the DMC contribution in the total $\mathrm{SAR}_{w b}$ may increase to $88 \%$, depending on the person's location in the considered indoor environment. A validation of the methodology can be found in Bamba et al (2013a). The human exposure to the diffuse fields has not yet been investigated. It is of utmost importance to account for the recent advances made in the wireless propagation literature in the assessment of the human whole-body $\mathrm{SAR}_{w b}$. Because of its stochastic nature, the numerical investigation of the whole-body $\mathrm{SAR}_{w b}$ due to diffuse fields would require excessive time and memory resources. The (fast) estimation of the average whole-body $\mathrm{SAR}_{w b}$ due to diffuse fields is also important for the dosimetry community as it could help for the revision of the current basic restrictions (ICNIRP 1998), which were derived from plane wave analysis (deterministic discrete plane wave exposure) neglecting the DMC.

The human absorption in the $\mathrm{GHz}$ region is mainly influenced by the body surface area (BSA). This is due to the absorption occurring at the body surface rather than inside the body volume because of the relative small penetration depth in the $\mathrm{GHz}$ region (Hirata et al 2007, Uusitupa et al 2010). Based on those findings, a BSA based formula to determine the $\mathrm{SAR}_{w b}$ caused by diffuse fields in the $\mathrm{GHz}$ region - from $1.45 \mathrm{GHz}$ up to $5.8 \mathrm{GHz}$ - is proposed.

The objective of this paper is to propose a simple formula with only the human mass and the power densities as inputs to determine rapidly the average whole-body $\mathrm{SAR}_{w b}$ under realistic propagation conditions, i.e., presence of diffuse fields and a possible LOS component. The novelty of this paper is that $i$ ) it proposes a formula whose parameters are independent of the human morphology to determine the $\mathrm{SAR}_{w b}$ ii) the formula includes the diffuse fields contribution in the human $\mathrm{SAR}_{w b}$ assessment. The theoretical background is based on an ellipsoid analysis, which is used to investigate the parameters involved in the proposed formula. For the LOS exposure, both vertical and horizontal polarization are investigated, and all azimuthal angles as well. Some parameters in the formula are frequency dependent and are characterized at $\mathrm{GHz}$ frequencies. Moreover, the proposed formula is validated using numerical simulations with several realistic 3D heterogeneous phantoms exposed at the frequency of $3 \mathrm{GHz}$.

The remainder of the paper is organized as follows. In Section 2, the human models, the simulation settings, and the methodology are described. The parameters involved in the formula are described in Section 3, as well as the proposed formula. The formula is further validated at $3 \mathrm{GHz}$ in Section 4. Further discussions and analysis of these results can be found in Section 4. We show in Section 5 how the formula can be applied to the data from measurements performed in an office building. Further discussions and advantages of the formula are highlighted in that Section. Finally, conclusions are drawn in Section 6. 


\section{Models, Settings and Methodology}

\subsection{Models}

The complexity of the human morphology makes a geometrical investigation very difficult for the $\mathrm{SAR}_{w b}$ determination. Prolate spheroids models are often used as approximation of the human body (Vermeeren et al 2008). The authors in that paper investigated the $\mathrm{SAR}_{w b}$ in the $\mathrm{MHz}$ region, where the dielectric properties influence more the $\mathrm{SAR}_{w b}$ than the BSA does. An ellipsoid model however, describes more accurately the human body characteristics in terms of the BSA (Conil et al 2011). Ellipsoids are therefore more suitable as non-realistic phantom for the determination of the $\mathrm{SAR}_{w b}$ in the $\mathrm{GHz}$ region. Five homogeneous ellipsoidal models of human bodies are used here, i.e., the average man, the average woman, the 10-year old child, and the 5-year old child to determine the main parameters in the $\mathrm{SAR}_{w b}$ prediction-equation. The mass (resp. height) of each ellipsoid coincides with the mass (resp. height) of the corresponding human model. A picture of the tri-axial ellipsoid model is shown in Figure 1. The three axes are denoted as $a, b$, and $c$ with $a \geq b \geq c$.

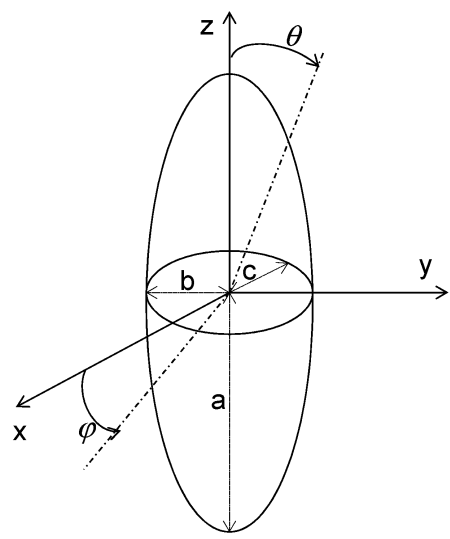

Figure 1. An ellipsoid model for human body approximation. $a, b$ and $c$ are the ellipsoids axes. $\varphi$ and $\theta$ represent the azimuth and the elevation, respectively.

The parameters of the ellipsoids, i.e., the height, length, and width are summarized in Table 1 and are retrieved from Durney et al (1986).

The dielectric properties (conductivity $\sigma$ and relative permittivity $\epsilon_{r}$ ) of equivalent head tissue recommended by the IEEE SCC-34/SC-2 (IEEE SCC 2003) are used to simulate the human tissues properties in the homogeneous phantoms. Their values for different frequencies in the $\mathrm{GHz}$ region are listed in Table 2.

Moreover, four 3D heterogeneous human models are used to validate the theory developed with the ellipsoid analysis. The four anatomical phantoms are the virtual family models (Thelonious, Billie, Ella, and Duke). Each model contains more than 75 different types of tissues and organs (ITIS 2012). A picture of the virtual family is shown in Fig. 2. 


\begin{tabular}{|l|l|l|l|l|}
\hline Phantoms & $\begin{array}{l}\text { Average } \\
\text { mass }(\mathrm{kg})\end{array}$ & $\begin{array}{l}\text { Height, } \\
2 a(\mathrm{~m})\end{array}$ & $\begin{array}{l}\text { Length, } \\
2 b(\mathrm{~m})\end{array}$ & $\begin{array}{l}\text { Width, } \\
2 c(\mathrm{~m})\end{array}$ \\
\hline $\begin{array}{l}\text { Average } \\
\text { man }\end{array}$ & 70.00 & $2 \times 0.875$ & $2 \times 0.195$ & $2 \times 0.098$ \\
\hline $\begin{array}{l}\text { Average } \\
\text { woman }\end{array}$ & 61.14 & $2 \times 0.805$ & $2 \times 0.200$ & $2 \times 0.091$ \\
\hline $\begin{array}{l}\text { 10-year-old } \\
\text { child }\end{array}$ & 32.20 & $2 \times 0.69$ & $2 \times 0.143$ & $2 \times 0.078$ \\
\hline $\begin{array}{l}\text { 5-year-old } \\
\text { child }\end{array}$ & 19.50 & $2 \times 0.56$ & $2 \times 0.120$ & $2 \times 0.069$ \\
\hline
\end{tabular}

Table 1. Characteristics of the ellipsoids used as human phantoms

\begin{tabular}{|c|c|c|}
\hline Frequency $(\mathrm{MHz})$ & \multicolumn{2}{|c|}{ Head tissue properties } \\
\hline & $\epsilon_{r}$ & $\sigma(\mathrm{S} / \mathrm{m})$ \\
\hline 1450 & 40.50 & 1.20 \\
\hline 1610 & 40.30 & 1.29 \\
\hline $1800-2000$ & 40.00 & 1.40 \\
\hline 2450 & 39.20 & 1.80 \\
\hline 3000 & 38.50 & 2.40 \\
\hline 5800 & 35.30 & 5.27 \\
\hline
\end{tabular}

Table 2. Head tissue dielectric parameters

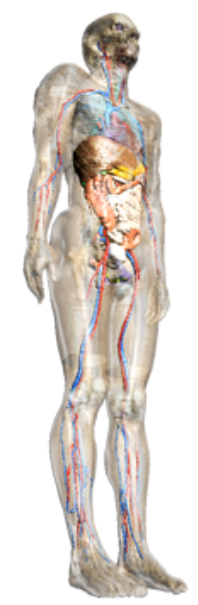

(a) Duke (34year-old male)

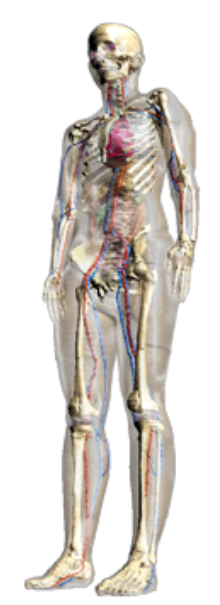

(b)

(26-year-old female)

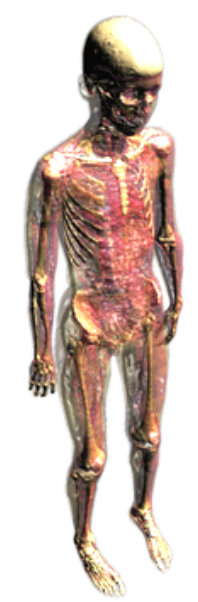

(c) Billie (11year-old girl)

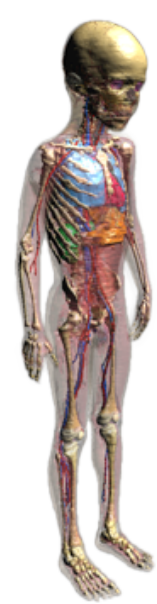

(d) Thelonious (6-year-old boy)

Figure 2. Various considered 3D heterogeneous human models

\subsection{Simulation Settings}

The Finite-Difference in Time-Domain (FDTD) method (Taflove et al 2005) is used to numerically compute the $\mathrm{SAR}_{w b}$ in the homogeneous ellipsoids and in the heterogeneous 
human models. A harmonic excitation and a rectangular plane wave source based on the total-field scattered-field (TFSF) technique is used in the simulations (Merewether et al 1980). The maximum grid step in the phantoms is set to $0.07 \lambda_{p h}$, where $\lambda_{p h}$ is the wavelength inside a tissue. An uniaxial perfectly matched layer (UPML) is used as boundary condition to avoid the reflections of the waves impinging on the boundaries of the simulation domain. The UPML is set so that more than $95 \%$ of the incident plane-wave power is absorbed by the boundary layers.

\subsection{Methodology}

While assessing experimentally the $\mathrm{SAR}_{w b}$ using the method of Bamba et al (2012), one of the challenging issues is the determination of the ACS under a LOS illumination $\left(\mathrm{ACS}_{l o s}\right)$. Bamba et al (2012) assumed a frontal illumination $(\varphi=0)$ or a back illumination $(\varphi= \pm \pi)$ of humans to determine roughly the $\mathrm{ACS}_{\text {los }}$ as half of the diffuse absorption cross section $\left(\mathrm{ACS}_{d m c}\right)$. In general, and for a given azimuth:

$$
A C S_{\text {los }}=k \times A C S_{d m c} \quad \text { with } 0<k<1,
$$

$\mathrm{ACS}_{d m c}$ is the whole-body ACS in diffuse fields, i.e., accounting fot all the azimuthal angles. On the one hand, the numerical approach can determine exactly the $\mathrm{ACS}_{\text {los }}$ given a certain incident angle. On the other hand, the $\mathrm{ACS}_{d m c}$ - which is determined in diffuse fields - can not be determined exactly via numerical simulations. The diffuse absorption cross section is therefore investigated from the ellipsoid analysis, and then related to the $\mathrm{ACS}_{l o s}$ via the parameter $k$.

The $\mathrm{SAR}_{w b}$ is the power absorbed by a human per unit of mass $(\mathrm{W} / \mathrm{kg})$. The SAR can be expressed as:

$$
\begin{aligned}
S A R_{w b} & =\frac{P_{a b s}}{m} \\
& =\frac{I \times A C S}{m}
\end{aligned}
$$

where $\mathrm{P}_{a b s}, \mathrm{~m}, \mathrm{I}$, and ACS are the absorbed power by the human (Watt), the person's mass $(\mathrm{kg})$, the incident power density $\left(\mathrm{Watt} / \mathrm{m}^{2}\right.$ ), and the person's absorption cross section $\left(\mathrm{m}^{2}\right)$, respectively.

The ACS results from the interaction between a surface area and a plane wave. It can therefore be defined as the projected surface (on a plane) scaled by an efficiency factor, which is the percentage of the power absorbed by the surface (Andersen et al 2007). All the wave interactions with the surface such as the creeping wave effects (if they occurs) are included in the efficiency factor. The human $\mathrm{ACS}_{d m c}$ can be defined as the sum of the different projected human body surfaces from all the azimuthal angles, and then scaled with the average fraction of the power absorbed by the human.

$$
\begin{aligned}
A C S_{d m c} & =\eta \times \int_{0}^{2 \pi} B S A_{p r}(\varphi) d \varphi \\
& =\eta \times B S A_{p r}^{T}
\end{aligned}
$$


where $\eta, \operatorname{BSA}_{p r}(\varphi)$, and $\mathrm{BSA}_{p r}^{T}$ are the average fraction of the incident power being absorbed, the projected surface $\left(\mathrm{m}^{2}\right)$ of the human from the azimuth $\varphi$, and the total projected body surface area $\left(\mathrm{m}^{2}\right)$ of the human accounting for all the azimuthal angles. Since $\eta$ is an average value, it is independent on the direction of incidence of the incoming wave.

2.3.1. Investigating the ellipsoid surface: Consider the ellipsoid of Figure 1. If the ellipsoid is fixed, its projection on a plane in the frontal direction $(\varphi=0$ and $z=0)$ is an ellipse whose long and small axis are $2 a$ and $2 b$, respectively. The surface area of that ellipse is $S_{F}=\pi \times a \times b$. Similarly, the area of the projected ellipsoid on a plane from a side view $\left(\varphi= \pm \frac{\pi}{2}\right.$ and $\left.z=0\right)$ is $S_{S}=\pi \times a \times c$. Suppose now that the projection of the ellipsoid is done on any plane between the front and the side view $\left(0<|\varphi|<\frac{\pi}{2}\right.$ and $z=0$ ), the projected surface is an ellipse whose long and small axis are $2 a$ and $h$, respectively. $h$ is the hypotenuse of the triangle whose perpendicular sides are $2 b \cos (\varphi)$ and $2 \operatorname{csin}(\varphi)$. The area of that surface is denoted as $\mathrm{S}_{\varphi}$, and is defined as follows:

$$
\begin{aligned}
S_{\varphi} & =\pi a \frac{h}{2} \\
& =\pi \frac{a}{2} \sqrt{(2 b \cos (\varphi))^{2}+(2 c \sin (\varphi))^{2}} \\
& =\sqrt{S_{F}^{2} \cos ^{2}(\varphi)+S_{S}^{2} \sin ^{2}(\varphi)}
\end{aligned}
$$

Equation (4) shows that the surface of the projected ellipsoid from any azimuthal angle can be described with the frontal and the side projected surfaces, i.e., $\left(\mathrm{S}_{F}\right)$ and $\left(\mathrm{S}_{S}\right)$, respectively. Analogously with (4), the ACS at any azimuth i.e., $\operatorname{ACS}(\varphi)$, can therefore be expressed as a function of the frontal and side absorption cross section, denoted as $\mathrm{ACS}_{F}$ and $\mathrm{ACS}_{S}$, respectively.

$$
A C S(\varphi)=\sqrt{A C S_{F}^{2} \cos ^{2}(\varphi)+A C S_{S}^{2} \sin ^{2}(\varphi)}
$$

The frontal $\left(\mathrm{ACS}_{F}\right)$ and the side $\left(\mathrm{ACS}_{S}\right)$ absorption cross section can be easily determined via FDTD numerical simulations and using (2b).

Before we derive the formula for the $\mathrm{SAR}_{w b}$, we recall an important property of the diffuse fields. We assume that the electromagnetic waves composing the diffuse fields are mutually incoherent, i.e., they cannot interfere with each other. Their energy can therefore be summed. This assumption is made for the acoustic case in Kuttruff et al (2000). The applicability of the acoustic diffuse fields properties to the electromagnetic diffuse fields is demonstrated in Andersen et al (2007).

The diffuse absorption cross section, which is our main concern is therefore expressed as:

$$
A C S_{d m c}=\int_{0}^{2 \pi} A C S(\varphi) d \varphi
$$

The expression in (6) is a $\frac{\pi}{2}$-periodic function; it can therefore be re-written as (using 
$5)$ :

$$
\begin{aligned}
A C S_{d m c} & =4 \int_{0}^{\frac{\pi}{2}} \sqrt{A C S_{F}^{2} \cos ^{2}(\varphi)+A C S_{S}^{2} \sin ^{2}(\varphi)} d \varphi \\
& =4 A C S_{F} \int_{0}^{\frac{\pi}{2}} \sqrt{1-m^{2} \sin ^{2}(\varphi)} d \varphi
\end{aligned}
$$

the integral in (7) is the complete elliptic integral of second order with the parameter $m$ defined as:

$$
m=\sqrt{\frac{A C S_{F}^{2}-A C S_{S}^{2}}{A C S_{F}^{2}}}, \quad 0<\mathrm{m}<1
$$

Equation (7) is used to determine the $\eta$ parameter expressed in (3). We assume that the diffuse field is completely random. If one would model the diffuse fields with a single plane wave, a polarization matching factor of 0.5 would be assumed, which corresponds to a polarization angle of $45^{\circ}$. Andersen et al (2012) for instance made such assumption. Both $\mathrm{ACS}_{F}$ and $\mathrm{ACS}_{S}$ are numerically determined with a plane wave incident to the front and to a side, respectively, with a polarization of $45^{\circ}$; which means that $\mathrm{ACS}_{F}$ and $\mathrm{ACS}_{S}$ are both obtained for $\eta$ averaged over the polarization cases. The resulting $\mathrm{ACS}_{d m c}$ value obtained here is therefore for the average $\eta$, and is independent on a particular polarization. Due to the symmetry of the ellipsoid, the same results would be achieved if we assumed a polarization of $\pm 135^{\circ}$ or $-45^{\circ}$.

Finally, the $\mathrm{SAR}_{w b}$ due to a LOS exposure depends on the incident plane wave azimuth, polarization, considered frequency, etc. (ICNIRP 1998, Uusitupa et al 2010). By analyzing (2a) and (2b), it is clear that the resulting $\mathrm{ACS}_{\text {los }}$ will depend on the parameters mentioned above. The idea is to gather all these dependencies in a limited number of parameters, i.e., $\eta$, and $k$. Once these parameters are characterized for a given posture, polarization, direction, and frequency range, the $\mathrm{ACS}_{d m c}$ and the $\mathrm{ACS}_{l o s}$ can be linked to the BSA. The upright posture is considered throughout this paper.

\section{Formula for human whole-body $\mathbf{S A R}_{w b}$}

\subsection{Determination of the parameters}

This section is devoted to the determination - through FDTD numerical simulations of the parameters $k$ and $\eta$ expressed in (1) and (3), respectively, for frequencies ranging from $1450 \mathrm{MHz}$ to $5800 \mathrm{MHz}$. These frequencies span from the Global System for Mobile Communications (GSM) 1800 band up to the Wireless Local Area Network (WLAN) frequencies. At each frequency, the ellipsoidal models are assigned with the appropriate dielectric properties listed in Table 2. In all the simulations, the incident plane wave power density is set to $1 \mathrm{~W} / \mathrm{m}^{2}$.

3.1.1. Validation of the ACS as a function of the azimuthal angle: The ACS expression of (5) at any azimuth as a function of the side and frontal ACS is validated here. All 
the plane waves are incident in the transverse plane. The azimuth angle is varied with a step of $15^{\circ}$, and both vertical and horizontal polarizations are investigated. Table 3 compares the theoretical $\mathrm{ACS}_{t h}$ (ACS using (5)) with the simulated $\mathrm{ACS}_{\text {sim }}$ (ACS using (2b)) from $\varphi=0$ to $\varphi=\frac{\pi}{2}$ (symmetry of the ellipsoid) for the average woman. For the sake of the paper length, only the results of the average woman are shown. The trend is however the same for the other phantoms. The maximum relative error $(\delta \mathrm{ACS})$ for all

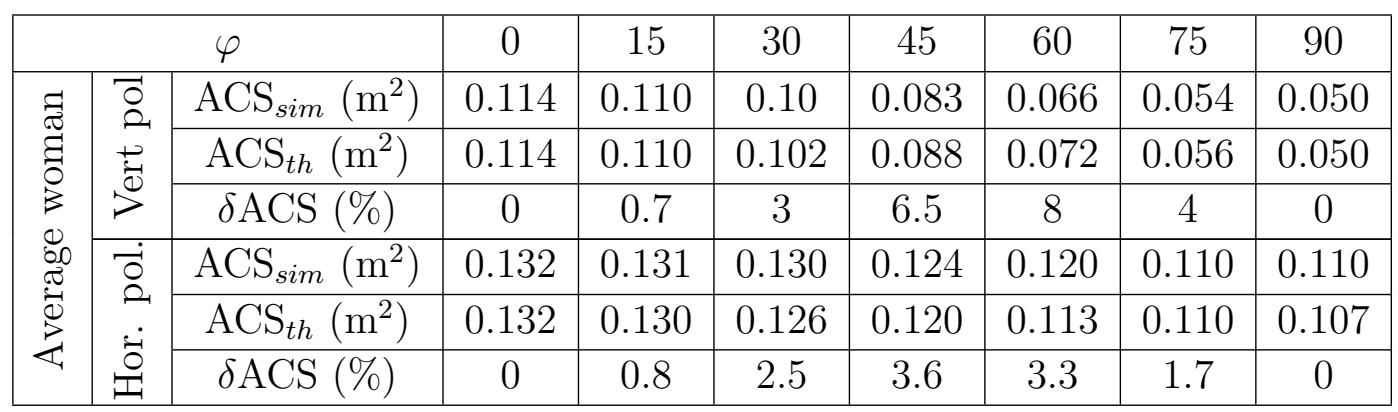

Table 3. Simulated $\left(\mathrm{ACS}_{\text {sim }}\right)$ and calculated $\left(\mathrm{ACS}_{t h}\right)$ absorption cross sections for the average woman. Both vertical and horizontal polarization at different azimuth angles are considered in the transverse plane.

the ellipsoids is about $8 \%$ (Average woman), whereas the minimum error approaches $0 \%$ ( 5 years old child). Given the small relative error between the predicted ACS with (5) and the simulated values for all the phantoms, (7) can therefore be used to determine the $\mathrm{ACS}_{d m c}$ of the ellipsoidal models in order to derive the $\eta$ parameter.

3.1.2. Determination of $\eta$ : Let us recall that $\eta$ is the ratio between the $\mathrm{ACS}_{d m c}$ and the total projected body surface area $\left(\mathrm{BSA}_{p r}^{T}\right)$. The $\mathrm{ACS}_{d m c}$ of an ellipsoid is determined with $(7)$ and few simulations. The projected surface area of the tri-axial ellipsoid can be found by integrating (4) from 0 to $2 \pi$, which leads to a similar expression as (7). The $\mathrm{ACS}_{d m c}$ is determined for all the investigated frequencies while the $\mathrm{BSA}_{p r}^{T}$ is frequency independent. For each frequency, four $\eta$ values - one value per phantom - are obtained, and then averaged to obtain one $\eta$ value per frequency. It turns out that $\eta$ is phantom independent at a given frequency since the maximum relative difference from the mean value is about $6 \%$ and this occurs at $5800 \mathrm{MHz}$. The average values of $\eta$ as a function of the frequency are shown in Figure 3. A linear regression is applied to the data of Figure 3, and $\eta$ can be expressed as a function of the frequency:

$$
\eta(f)=-1.7827 \times 10^{-5} \times f+0.5859, \quad R^{2}=0.9981
$$

where $f$ is the frequency in $\mathrm{MHz}$, and $\mathrm{R}^{2}$ is the coefficient of determination. Theoretically, $(8)$ is valid $(0<\eta(f)<1)$ up to $32.80 \mathrm{GHz}$.

3.1.3. Determination of the $k$-parameter: The next parameter to investigate is the ratio $k$ between the $\mathrm{ACS}_{\text {los }}$ and the $\mathrm{ACS}_{d m c}$. This parameter is needed to determine 


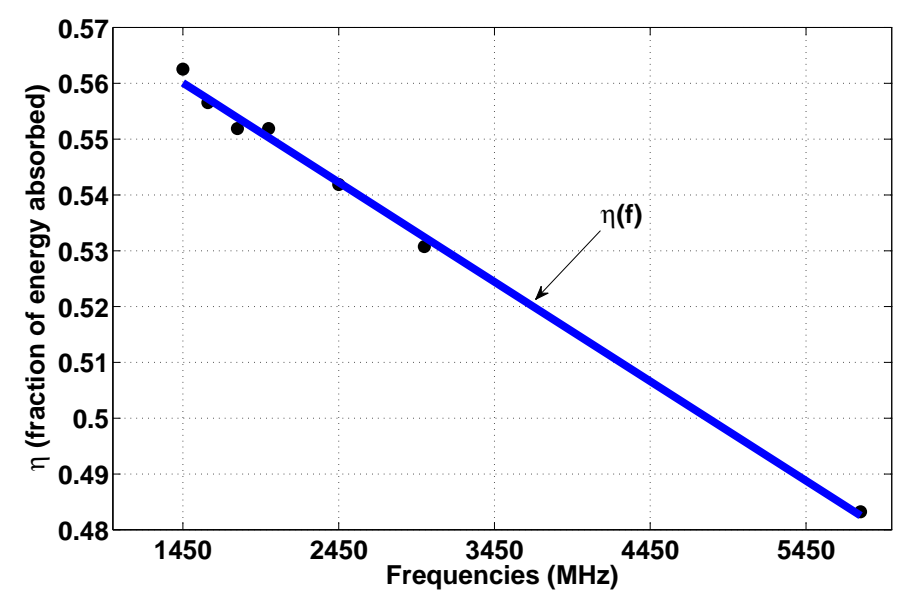

Figure 3. $\eta$-parameter as a function of the frequency

the $\mathrm{SAR}_{w b}$ due to a LOS exposure. The $\mathrm{ACS}_{\text {los }}$ depends obviously on the incident wave polarization, azimuthal angle, etc. The parameter $k$ is therefore expressed as:

$$
k(\varphi, \psi)=\frac{A C S_{l o s}(\varphi, \psi)}{A C S_{d m c}}
$$

The $k$-parameter is investigated using the four ellipsoids (average man, average woman, 10 years old, and 5 years old), for the vertical and horizontal polarization. The azimuthal angle is varied from 0 to $90^{\circ}$ with a step of $15^{\circ}$ (azimuthal symmetry of the ellipsoid). For a given frequency and azimuthal angle, the $k$-parameter is averaged over the four phantoms. A frequency averaging is also done for each azimuthal angle. For all the investigated frequencies and azimuthal angles, a maximum relative standard deviation of about $3 \%\left(\varphi=90^{\circ}\right.$, frequency $\left.=2450 \mathrm{MHz}\right)$ and $1.5 \%\left(\varphi=0^{\circ}\right.$, frequency $\left.=1450 \mathrm{MHz}\right)$ is obtained for the vertical and horizontal polarization, respectively. The $k$-parameter can therefore also be considered as phantom independent. Figure 4 shows the mean value of the $k$-parameter for the vertical $\left(k_{V}\right)$ and horizontal $\left(k_{H}\right)$ polarization. Again a small maximum relative standard deviation of about $2 \%\left(\varphi=90^{\circ}\right)$ and $2 \%\left(\varphi=0^{\circ}\right)$ is obtained for the vertical and horizontal polarization, respectively. It is observed that $k_{H}$ ( $k$ value for the horizontal polarization) is always - as a function of the azimuth - higher than $k_{V}$ ( $k$ value for the vertical polarization). This is an expected result since the $\mathrm{SAR}_{w b}$ from a horizontally polarized plane wave is higher than the one from a vertically polarized plane wave in the GHz region (Uusitupa et al 2010). Given the low relative standard deviations, the $k$-parameter can therefore be considered as phantom and frequency independent. The $k$-parameter for any other polarization $\psi$ can be easily determined with the knowledge of $k_{V}$ and $k_{H}$.

$$
k(\psi)=k_{V} \times \cos ^{2}(\psi)+k_{H} \times \sin ^{2}(\psi)
$$




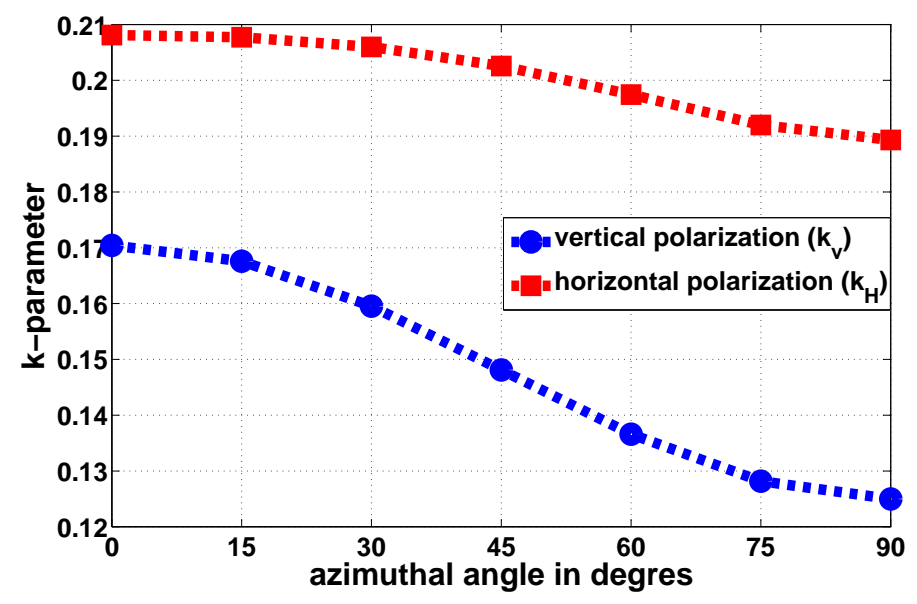

Figure 4. $k$-parameter (averaged over the phantoms and the frequencies). $\mathrm{k}_{V}$ (resp. $\mathrm{k}_{H}$ ) is the ratio between the ACS from a vertically (horizontally) polarized LOS and the DMC.

\subsection{Experimental determination of the diffuse ACS for the realistic human models}

The $\mathrm{ACS}_{d m c}$ is calculated with the knowledge of the phantom $\mathrm{BSA}_{p r}^{T}$ (total projected BSA) and the parameter $\eta$, see (3). However, the human BSA is very complex, depending mainly on the height and the mass. Several works (Du Bois et al 1916, Fujimoto et al 1968, Gehan et al 1970) have investigated the human BSA and expressed it with equations using both the human height and mass. All these equations determine accurately the human BSA, but they are unnecessarily complex and in addition they are inaccurate for obese humans (Livingston et al 2001). Actually, the human BSA can be determined with the mass as the only input and the formula gives a better estimation of the BSA for both normal and obese people (Livingston et al 2001). A human BSA is expressed as follows (Livingston et al 2001):

$$
B S A=0.1173 \times m^{0.6466}, \quad \text { if } \mathrm{m} \geq 10 \mathrm{~kg}
$$

where BSA is the human body surface area in $\mathrm{m}^{2}$ and $\mathrm{m}$ stands for the human mass in $\mathrm{kg}$. Livingston et al (2001) derived this formula from patients weighting from $51.3 \mathrm{~kg}$ to $248.6 \mathrm{~kg}$ (their heights ranged from $152 \mathrm{~cm}$ to $182 \mathrm{~cm}$ ), which means that the child models were not included in their procedure. Moreover, (11) was derived based on a geometric method along with the body size scaling principles. The surface area of a body segment (arm, hand, leg, thigh, etc.) is estimated by multiplying its circumference by its length and by a factor correcting for the shape.

The formula, i.e., (11) is important for this study. We therefore investigate its correctness via numerical simulations and adapt it if necessary.

Numerical determination of the body surface area and total projected body surface area of heterogeneous phantoms: One can determine numerically the BSA ( $\left.\mathrm{BSA}_{\text {num }}\right)$ of a phantom in SEMCAD-X. This is achieved by discretizing the phantom with a certain 
uniform grid (voxeling), and by taking the sum of the surfaces of the voxels which are adjacent to the air. For this calculation, a grid step of $5 \mathrm{~mm}$ was chosen. The procedure to determine numerically the heterogeneous phantom BSA is explained in Appendix A. Table 4 compares the BSA values of the heterogeneous phantoms determined with (11) and those determined numerically $\left(\mathrm{BSA}_{\text {num }}\right)$. The numerical simulation results in

\begin{tabular}{|c|c|c|c|c|}
\hline Phantom & Thelonious & Billie & Ella & Duke \\
\hline $\mathrm{BSA}_{\text {num }}\left(\mathrm{m}^{2}\right)$ & 0.640 & 0.976 & 1.350 & 1.570 \\
\hline $\mathrm{BSA}\left(\mathrm{m}^{2}\right)$ & 0.798 & 1.180 & 1.622 & 1.878 \\
\hline $\mathrm{BSA}^{2} \mathrm{BSA}_{\text {num }}$ & 1.24 & 1.21 & 1.20 & 1.20 \\
\hline
\end{tabular}

Table 4. Ratio between the body surface area values, i.e., one determine numerically and the other determine with (11).

Table 4 show that (11) overestimates the BSA of all the phantoms. The overestimation is constant for all the phantoms and is about $20 \%$, showing that the formula (11) approximates well the human body surface area. However, we correct this formula with the $20 \%$ overestimation to obtain more accurate estimation of the body surface area. The body surface area estimation formula is then as follows:

$$
B S A=0.097 \times m^{0.6466},
$$

which agrees well with the numerical results. The formula is mainly interesting because it determines accurately the BSA of any human based solely on its mass.

The body surface area is aimed to calculate the $\mathrm{ACS}_{d m c}$ of heterogeneous humans via the parameter $\eta$. However, it is important to notice that the BSA expressed by (12) can not be used to determine the $\mathrm{ACS}_{d m c}$ since it is different from the total projected BSA, namely the $B S A_{p r}^{T}$, see (3). Therefore, the BSA expressed with (12) needs to be corrected with a coefficient to obtain the $\mathrm{BSA}_{p r}^{T}$, assuming that both quantities have the same ratio for all the phantoms. Like for the body surface area, the total projected body surface area of the heterogeneous phantoms is determined here via numerical simulations. The procedure is described in Appendix B. Table 5 shows the numerical values of the BSA and $\mathrm{BSA}_{p r}^{T}$ for the four heterogeneous phantoms, along with their respective ratio. The average ratio is about 2.20 with a relative standard deviation of about

\begin{tabular}{|l|c|c|c|c|}
\hline Phantom & Thelonious & Billie & Ella & Duke \\
\hline $\mathrm{BSA}_{\text {num }}\left(\mathrm{m}^{2}\right)$ & 0.64 & 0.98 & 1.35 & 1.57 \\
\hline $\mathrm{BSA}_{\text {num }, p r}^{T}\left(\mathrm{~m}^{2}\right)$ & 1.45 & 2.17 & 2.90 & 3.35 \\
\hline $\mathrm{BSA}_{\text {num }, p r}^{T} / \mathrm{BSA}_{\text {num }}$ & 2.265 & 2.22 & 2.15 & 2.14 \\
\hline
\end{tabular}

Table 5. Numerical body surface area and total projected body surface area with their respective ratio.

2.70\%. This ratio should be used to obtain the $\mathrm{BSA}_{p r}^{T}$ from the BSA calculated with (12). 


\subsection{Formula for Whole-body $S A R_{w b}$ as a function of the human mass}

A person is assumed to be exposed to both LOS plane wave and to diffuse fields in a realistic environment according to the room electromagnetics theory (Andersen et al 2007, 2012).

The whole-body $\mathrm{SAR}_{w b}$ due to a LOS plane wave illumination is obtained by arranging (2b), (3), (9) and (12), and by accounting for the ratio between the BSA ${ }_{p r}^{T}$ and the BSA determined in Section 3.2.

$$
S A R_{w b, l o s}=0.21 \times m^{-0.3534} \times \eta \times k \times I_{l o s} \quad \mathrm{~m} \geq 10 \mathrm{~kg}
$$

where $\mathrm{I}_{\text {los }}$ is the incident power density of the LOS component.

In indoor environments, the $\mathrm{SAR}_{w b}$ due to the $\mathrm{DMC}$ is stochastic since the propagation of the diffuse fields is stochastic (Ullmo et al 1999). The diffuse fields properties (polarization and phase) are assumed to be random variables and will be further defined. For the average whole-body $\mathrm{SAR}_{a v g, d m c}$ due to diffuse fields, we arrange the same equations as for the $\mathrm{SAR}_{w b, l o s}$.

$$
S A R_{\text {avg }, d m c}=0.21 \times m^{-0.3534} \times \eta \times I_{d m c} \quad \mathrm{~m} \geq 10 \mathrm{~kg}
$$

Since the parameters $\mathrm{k}, \eta$, and the power densities are known, the average human absorption due to a LOS plane wave and/or the diffuse fields is easily calculated if the human mass is known.

\section{Validation of the $\mathbf{S A R}_{w b}$ formula}

The proposed formulas are validated through numerical simulations at the frequency of $3 \mathrm{GHz}$. The formula are applied to the 3D heterogeneous human models (see Fig. 2) and are compared to the $\mathrm{SAR}_{w b}$ values obtained from the FDTD numerical simulations. The $\mathrm{SAR}_{w b}$ due to the LOS component and the DMC are separately validated. The stochastic properties of the DMC are applied here. In both cases, the total incident power density is set to $1 \mathrm{~W} / \mathrm{m}^{2}$.

\section{1. $S A R_{w b}$ values from LOS plane wave exposure}

Accounting only the LOS exposure, the $\operatorname{SAR}_{w b, l o s}(\varphi, \psi)$ due to a LOS plane wave with a polarization $\psi$ and azimuth $\varphi$ is expressed as (13) with $\mathrm{k}(\varphi, \psi)$ instead of $\mathrm{k}$, see Fig. 4 . The theoretical $\mathrm{SAR}_{w b, l o s}^{t h}$ determined with (13) versus the simulated $\mathrm{SAR}_{w b, l o s}^{\text {sim }}$ from the numerical simulations for Ella is shown in Fig. 5. In accordance with the literature (Hirata et al 2009, Uusitupa et al 2010) and the simulated values, the developed method shows that the $\mathrm{SAR}_{w b}$ induced by a horizontally polarized plane wave is always higher - with respect to the azimuth - than the $\mathrm{SAR}_{w b}$ induced by a vertically polarized plane wave (see Fig. 5(a)). We also notice that the $\mathrm{SAR}_{w b, l o s}$ is more sensitive to the azimuth 


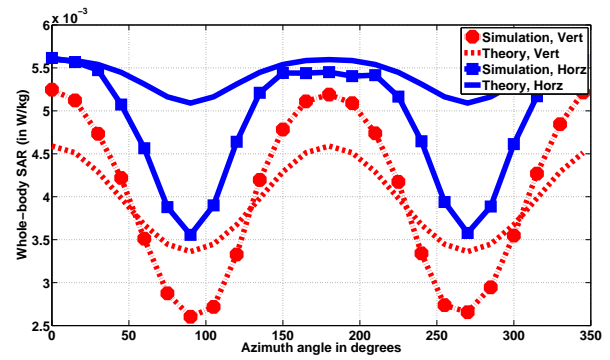

(a) $\mathrm{SAR}_{w b}$ values

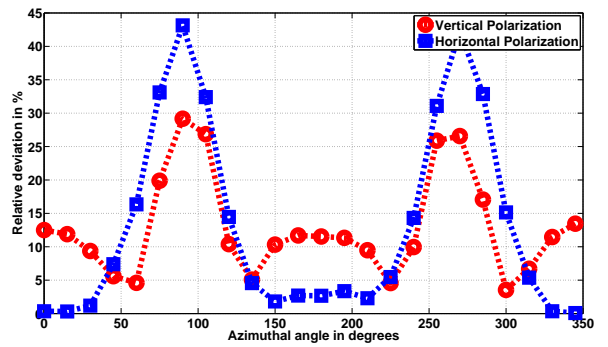

(b) Relative error of the $\mathrm{SAR}_{w b}$ with respect to simulations

Figure 5. Theoretical versus simulated $\mathrm{SAR}_{w b, l o s}$ and the relative error for Ella

\begin{tabular}{|l|l|l|l|l|l|l|l|l|}
\hline Phantom & \multicolumn{2}{|c|}{ Thelonious } & \multicolumn{2}{c|}{ Billie } & \multicolumn{2}{c|}{ Ella } & \multicolumn{2}{c|}{ Duke } \\
\hline Polarization & Vert. & Horiz. & Vert. & Horiz. & Vert. & Horiz. & Vert. & Horiz. \\
\hline$\delta \mathrm{SAR}_{\text {max }}(\%)$ & 39.80 & 25.30 & 30.30 & 19.40 & 29.20 & 43.10 & 36.10 & 40.40 \\
\hline$\delta \mathrm{SAR}_{\text {min }}(\%)$ & 13.00 & 0.10 & 3.00 & 2.00 & 3.50 & 0 & 2.40 & 1.20 \\
\hline$\delta \mathrm{SAR}_{\text {mean }}(\%)$ & 28.70 & 14.30 & 19.40 & 12.30 & 12.90 & 13.00 & 14.00 & 14.60 \\
\hline
\end{tabular}

Table 6. Relative error between the formula based $\mathrm{SAR}_{w b, l o s}$ and the simulation based $\mathrm{SAR}_{w b, l o s}$.

when the incident LOS wave is vertically polarized, which has been reported in Uusitupa et al (2010). All these remarks obtained for Ella are valid for the other members of the virtual family.

Table 6 summarizes the performance (i.e., the accuracy) - in terms of relative errors - of the formula applied to the other members of the virtual family. For convenience, we denoted by vertical (resp. horizontal) relative error the relative error between the $\mathrm{SAR}_{w b, l o s}^{t h}$ value obtained from (13) and from the simulated $\mathrm{SAR}_{w b, l o s}^{\text {sim }}$ value when the incident plane wave is vertically (resp. horizontally) polarized.

The mean vertical (resp. horizontal) relative error varies from $28 \%$ to $12 \%$ (resp. $14 \%$ to $12 \%$ ). The child models (Thelonious and Billie) exhibit in general the highest relative errors when the plane wave is incident to the front and/or the back $\left(\varphi \approx 0^{\circ}, 180^{\circ}\right)$ whereas the lowest errors are obtained when the wave is incident to the sides $\left(\varphi \approx 90^{\circ}\right.$, $270^{\circ}$ ). Regarding the adult models (Ella and Duke), it is interesting to see that the lowest errors occur for the worst case exposure, i.e., a horizontally polarized plane wave with frontal or dorsal incidence.

In general, the accuracy - in terms of relative error - of the proposed formula varies with the phantom mass as well. It is noteworthy to mention that the numerical $\mathrm{SAR}_{w b}$ values may vary due to small changes in the modeling of the parameters such as the model discretization, the absorbing boundaries conditions, the tissues properties, etc. A sensitivity study in Bakker et al (2010) shows that the uncertainty over the simulated $\mathrm{SAR}_{w b}$ may reach $21 \%$. Keeping the uncertainty issues in mind, the proposed formula gives a good estimation of the $\mathrm{SAR}_{w b}$ due to a single plane wave for increasing human 
model mass.

\subsection{Averaged $S A R_{w b, d m c}$ due to diffuse fields}

As mentioned above, the whole-body $\mathrm{SAR}_{w b}$ due to diffuse fields is statistically addressed. The reason of this approach is that modeling the object's position, the wall features and other details influencing the diffuse scattering fields propagation in indoor environments are extremely difficult - if not impossible - to obtain. The stochastic approach of the diffuse scattering fields has been widely accepted in the literature (Saleh et al 1987, Andersen et al 1995, Ullmo et al 1999, Chizhik et al 2008, Tanghe et al 2011). Vermeeren et al (2008) developed a statistical multipath exposure tool - which is based on FDTD simulations - to determine the average whole-body $\mathrm{SAR}_{w b}$ in realistic environments. We use this statistical multipath exposure method to validate the average whole-body $\mathrm{SAR}_{a v g, d m c}$ due to diffuse fields and calculated with (14). Vermeeren et al (2008) required a minimum of 4000 samples to obtain statistically relevant values.

In our numerical modeling, each sample is comprised of 432 plane waves (72 in the azimuthal plane and 6 in the elevation plane) representing the diffuse fields. Let us define now the properties of the diffuse fields within a sample. The total power density

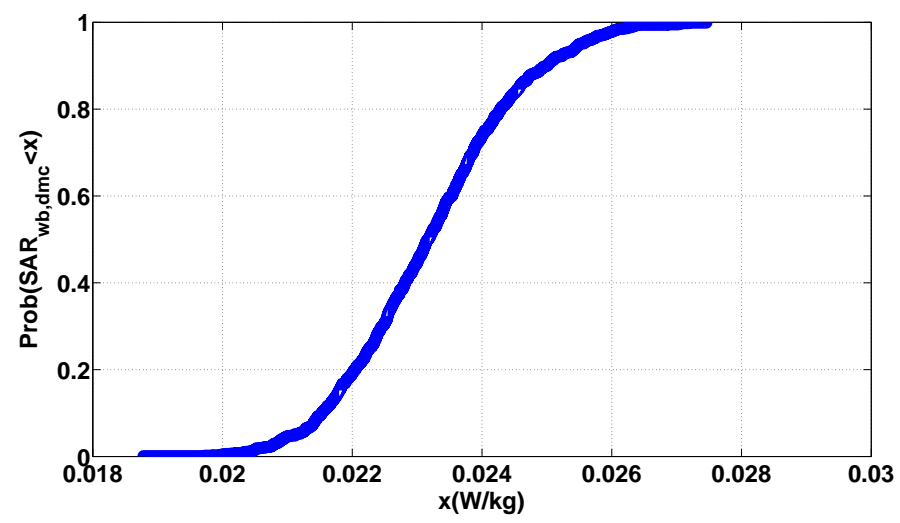

Figure 6. Empirical cumulative distribution of the $\mathrm{SAR}_{w b, d m c}$ for Duke

is divided by the number of plane waves in a sample such that each plane wave has the same amplitude (isotropic scattering). The polarization of the diffuse fields is such that half of the total received power would be received by a vertically-polarized antenna and the other half by a horizontally-polarized antenna. Therefore, the mean or average polarization in diffuse fields is modeled with $\pi / 4$. In addition, the polarization of a plane wave in diffuse fields is random and may take any value in $[02 \pi$. Therefore, we model here the diffuse field's polarization in each sample with a normal distribution with its mean $\mu=\pi / 4$ and standard deviation $\sigma=\pi / 4$. The standard deviation is chosen so that the polarization of a plane wave will be drawn in $[-4 \sigma, 4 \sigma[$, which corresponds to $[02 \pi[$, as just mentioned just above.

Regarding the plane waves phase, it is important to mention that when the phase is 
randomly drawn with equal probability in the range $[02 \pi[$, the numerical results do not match the experimental ones when it comes to emulate the electromagnetic wave propagation in reverberation chambers (Primiani et al 2012), which emulate indoor environments where the diffuse fields are prominent. Here, the phase of each plane wave is randomly chosen with equal probability in the range $\left[\begin{array}{ll}0 & \pi\end{array}\right]$ (Hill et al 1995). In a sample, the 432 plane waves surround the heterogeneous human model with a separation of $5^{\circ}$ between adjacent plane waves in the azimuthal plane, and $30^{\circ}$ between adjacent plane waves in the elevation plane. The plane waves in a set are incident simultaneously on the considered phantom.

The output of the statistical tool is the cumulative distribution function (cdf) of the $\mathrm{SAR}_{d m c}$. As an example, Fig. 6 shows the cdf of the $\mathrm{SAR}_{d m c}$ for Duke. Since we are mainly interested in the average value for a given phantom, we summarize those values in Table 7, along with the average value obtained from the developed formula and the relative error $\delta \mathrm{SAR}_{w b, d m c}$.

\begin{tabular}{|c|c|c|c|c|}
\hline & Thelonious & Billie & Ella & Duke \\
\hline $\mathrm{SAR}_{w b, d m c}^{\text {formula }}(\mathrm{mW} / \mathrm{kg})$ & 41 & 33 & 27.60 & 25.70 \\
\hline $\mathrm{SAR}_{\text {avg }, \text { dmc }}^{\text {tool }}(\mathrm{mW} / \mathrm{kg})$ & 67.70 & 37.40 & 24.90 & 23.20 \\
\hline$\delta \mathrm{SAR}_{w b, d m c}(\%)$ & -39.40 & -11.70 & 10.70 & 10.60 \\
\hline
\end{tabular}

Table 7. Whole-body $\mathrm{SAR}_{w b}$ values from diffuse fields. Comparison of the proposed formula $\left(\mathrm{SAR}_{w b, d m c}^{\text {formula }}\right)$ versus the statistical tool $\left(\mathrm{SAR}_{a v g, d m c}^{\text {tool }}\right)$.

For the $\mathrm{SAR}_{a v g, d m c}$ a maximum relative error of about $-39.40 \%$ is obtained, and this occurs for Thelonious. A relative error of about $-11.70 \%, 10.70 \%$ and $10.60 \%$ is obtained for Billie, Ella and Duke, respectively, indicating good agreement with the statistical tool. Note that the proposed formula underestimates the $\mathrm{SAR}_{\text {avg,dmc }}$ for the child models while it overestimates the $\mathrm{SAR}_{a v g, d m c}$ for the adult models. However, satisfactory agreement is obtained in general, especially for the adults.

\section{Application to epidemiological research and discussion}

\subsection{Presentation of the measurements data}

We show in this section how the proposed formula can be applied to dosimetry for epidemiological research in realistic indoor environments. Once again, the main novelty of the formula resides in the fact that the diffuse fields are accounted for in the human whole-body $\mathrm{SAR}_{w b}$ caused by electromagnetic $\mathrm{RF}$ radiation in realistic environments. The measurement data of Verloock et al (2009) are used and we assume that electromagnetic reverberation occurs in the environment, i.e., the total recorded field is comprised of discrete components (refer to as LOS component) and diffuse fields (refer to as DMC). Verloock et al (2009) determined Wireless Local Area Network (WLAN) exposure in an office environment. The transmitting source (access point) was 
a D-Link AirPlus G+ Wireless Router $(802.11 \mathrm{~b} / \mathrm{g})$. The incident fields were recorded with a spectrum analyzer and averaged over 6 minutes as recommended in ICNIRP (1998).

As mentioned in the introduction, a spectrum analyzer records the total field but can not display the contribution of the diffuse scattered fields in the total power. However, the contribution of the diffuse fields to the total power involved in an indoor environment has been recently addressed (Poutanen et al 2011, Bamba et al 2013). We investigate here five scenarios taken from the Figure 5 of Bamba et al (2013). A scenario corresponds to a distance separating the user from the source and where the diffuse fields represent a certain fraction of the total field. Those scenarios have been determined in the same indoor office where Verloock et al (2009) have conducted their measurements. Table 8 lists the total RMS electric field (as a function of the distance) measured in Verloock et al (2009). The contribution of the DMC in each scenario is taken from Bamba et al (2013) and is reported as well. Using these contributions, we have calculated the power densities of both the LOS and DMC components, see Table 8. It is important to mention

\begin{tabular}{|l|c|c|c|c|c|c|}
\hline & $\begin{array}{c}\text { distance user- } \\
\text { source }\end{array}$ & $\begin{array}{c}\mathrm{E}_{\text {total }}^{\text {avg }}(\mathrm{mV} / \mathrm{m}) \\
\text { Scenario 1 }\end{array}$ & $\begin{array}{c}\text { Total power } \\
\text { density }\left(\mu \mathrm{W} / \mathrm{m}^{2}\right)\end{array}$ & $\begin{array}{c}\text { Contribution of } \\
\text { the DMC }\end{array}$ & $\begin{array}{c}\text { LOS power } \\
\text { density }\left(\mu \mathrm{W} / \mathrm{m}^{2}\right)\end{array}$ & $\begin{array}{c}\text { DMC power } \\
\text { density }\left(\mu \mathrm{W} / \mathrm{m}^{2}\right)\end{array}$ \\
\hline Scenario 2 & $1 \mathrm{~m}$ & 104 & 99 & $1 \%$ & 98 & 1 \\
\hline Scenario 3 & $2 \mathrm{~m}$ & 70 & 13 & $13 \%$ & 11.3 & 1.4 \\
\hline Scenario 4 & $3 \mathrm{~m}$ & 72 & 14 & $25 \%$ & 10.5 & 3.5 \\
\hline Scenario 5 & $4 \mathrm{~m}$ & 114 & 34.3 & $32 \%$ & 23.3 & 11 \\
\hline
\end{tabular}

Table 8. LOS and DMC exposure values in office environment. The $\mathrm{E}_{\text {total }}^{a v g}$ values are taken from Verloock et al (2009) and the contribution of the DMC in each scenario is taken from Bamba et al (2013).

that the various incidence angles of the incident fields may vary over the 6 minutes, and the polarization as well (the exposed subject may move or rotate during the exposure time). A spectrum analyzer can not display the incidence angle, nor the polarization angle of an incident E-field. These properties of the incident LOS component are usually stochastically addressed. For instance, Bolte et al (2011) assumed that the polarization of the incident field is uniformly distributed whereas other studies assume a Gaussian distribution (Vermeeren et al 2008, Thielens et al 2013). We do not aim to investigate these issues here as this is out of the scope of this study.

For a possible comparison with the ICNIRP basic restrictions, we determine the $\mathrm{SAR}_{w b}$ values under the worst-case exposure conditions, i.e., frontal/dorsal incidence with a horizontal polarization $\left(\mathrm{k}_{H}\right.$ with $\varphi=0^{\circ}$ in Fig. 4$)$.

\subsection{Determination of the whole-body $S A R_{w b}$ and discussions}

We apply the formula - (13) and (14) - to determine the averaged whole-body $\mathrm{SAR}_{w b}$ of five persons $\left(\mathrm{p}_{1}, \mathrm{p}_{2}, \mathrm{p}_{3}, \mathrm{p}_{4}\right.$, and $\left.\mathrm{p}_{5}\right)$ with their respective mass $\left(\mathrm{m}_{1}=20 \mathrm{~kg}, \mathrm{~m}_{2}=45 \mathrm{~kg}\right.$, 


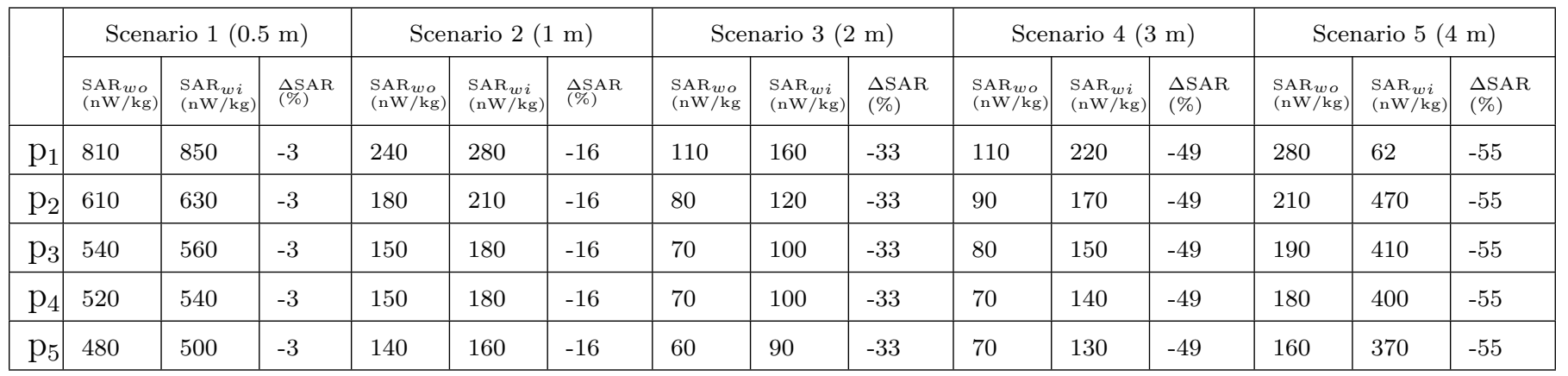

Table 9. Averaged whole-body $\mathrm{SAR}$ values. $\mathrm{SAR}_{w o}$ is the $\mathrm{SAR}_{w b}$ when the total power density is only comprised of specular components. $\mathrm{SAR}_{w i}$ is the $\mathrm{SAR}_{w b}$ when the presence of the DMC is accounted for. All the $\mathrm{SAR}_{w b}$ values are in $\mathrm{nW} / \mathrm{kg}$.

$\mathrm{m}_{3}=65 \mathrm{~kg}, \mathrm{~m}_{4}=70 \mathrm{~kg}$, and $\mathrm{m}_{5}=90 \mathrm{~kg}$ ). Knowing the operating frequency of WiFi, i.e., $\mathrm{f}_{0}=2.45 \mathrm{GHz}$, an average absorption coefficient $\eta$ of about 0.54 is obtained, see (8). The value of $k_{H}$ in (13) is about 0.20 and is obtained from Fig. 4. Applying (13) and (14) and the power densities measured with the spectrum analyzer in Table 8, one can readily determine the total average whole-body $\mathrm{SAR}_{w b}$ of our five subjects. Table 9 lists the $\mathrm{SAR}_{w b}$ values when the DMC contribution is not accounted for in the total power density $\left(\mathrm{SAR}_{w o}\right)$ and the $\mathrm{SAR}_{w b}$ values when the DMC contribution is accounted for $\left(\mathrm{SAR}_{w i}\right) . \quad \triangle \mathrm{SAR}$ is the relative error between the $\mathrm{SAR}_{w o}$ and $\mathrm{SAR}_{w i}$. $\mathrm{SAR}_{w o}$ is the whole-body SAR assuming that the total power density is comprised of only specular components (without the DMC), whereas $\mathrm{SAR}_{w i}$ is the whole-body SAR when the total power density is comprised of both specular components and diffuse fields. $\mathrm{SAR}_{w o}$ is calculated with the power density in the fourth column of Table 8 and $\mathrm{SAR}_{w i}$ is calculated with the power densities in the last two columns of Table 8. In either case, the total power density is the same for a given scenario.

The actual $\mathrm{SAR}_{w b}$ caused by electromagnetic radiation in the office building is underestimated when the DMC contribution is ignored in the total power density. The underestimation of the $\mathrm{SAR}_{w b, t o t a l}$ is about $-3 \%,-16 \%,-33 \%,-49 \%$, and $-55 \%$ for the scenario 1, 2, 3, 4, and 5, respectively. This clearly indicates that the contribution of the DMC must be accounted for in the assessment of the absorption in indoor environments, especially at larger distance.

The maximum (resp. minimum) $\mathrm{SAR}_{w b \text {,total }}$ (denoted as $\mathrm{SAR}_{w i}$ in Table 9) is obtained for the person with the smallest (resp. highest) mass, which is in agreement with the literature. The highest total absorption occurs for scenario 1 (500-805 nW/kg), followed by scenario $5(370-620 \mathrm{nW} / \mathrm{kg})$, scenario $2(160-280 \mathrm{nW} / \mathrm{kg})$, scenario 4 (130$220 \mathrm{nW} / \mathrm{kg})$, and scenario $3(90-160 \mathrm{nW} / \mathrm{kg})$, which is linearly dependent on the total power density. The relative error is higher at larger distance because the contribution of the DMC (due to diffractions, scattering) in the total power becomes prominent at larger distance (Bamba et al (2013)) and this induces a large error when it is not accounted in the absorption. The influence of the DMC on the measured fields - at larger distance - has been pointed out in Verloock et al (2009) and can be seen in the third column of 
Table 8.

The highest total electric field of $193.30 \mathrm{mV} / \mathrm{m}$ (scenario 1) was 300 times below the reference level of $61 \mathrm{~V} / \mathrm{m}$ for the general public. The maximum $\mathrm{SAR}_{w b, t o t a l}\left(\mathrm{p}_{1}\right.$ in scenario 1) is $10^{5}$ times below the basic restriction limit of $0.08 \mathrm{~W} / \mathrm{kg}$ for the general public.

The main advantage of the proposed formula is its ability to account for the diffuse fields in the human absorption. The formula is an alternative for numerical simulations of the $\mathrm{SAR}_{w b}$ caused by diffuse fields, which would be excessively demanding in terms of time and memory resources. Moreover, the formula's parameters are independent of the human size or morphology. Therefore, the formula is applicable to a population as long as their respective mass is known and useful for the epidemiological community. In this way, epidemiologists can account for whole-body absorption instead of only electric field strength values from personal exposimeter measurements as in Joseph et al (2008) and Röösli et al (2008).

\section{Conclusions}

Theoretical analysis of the ellipsoid and few simulations lead to a simple expression to determine the human $\mathrm{SAR}_{w b}$ under realistic conditions in the $\mathrm{GHz}$ region. The propagation model is based on the room electromagnetics model, i.e., existence of diffuse scattered fields and a possible LOS component. For an incident plane wave, both vertical and horizontal polarizations are considered and the method can easily be extended to other polarizations. The formula accounts for the exposure due to the diffuse multipath components, which may be prominent in realistic indoor environments. The strength of the formula resides in its simplicity because only the human mass and the power densities are needed. The proposed formula to determine the human $\mathrm{SAR}_{w b}$ has been validated with FDTD numerical simulations at $3 \mathrm{GHz}$, and good agreement is obtained for both LOS and diffuse illumination. The formula approximates the human $\mathrm{SAR}_{w b}$ caused by a LOS exposure with average relative errors varying from $28 \%$ to $12 \%$ for a vertical polarization (and from $14 \%$ to $12 \%$ for a horizontal polarization), depending on the phantom. For the diffuse exposure, relative errors of $-39.40 \%,-11.70 \%, 10.70 \%$ and $10.60 \%$ are obtained for the 6-year-old boy, 11-year-old girl, 26-year-old female, and 34-year-old male, respectively. The accuracy of the proposed formula improves when the human mass increases. Finally, the applicability of the formula is shown by applying it to personal dosimetry measurements for studies in an office building.

The accuracy of the formula to determine the $\mathrm{SAR}_{w b}$ due to a LOS exposure at certain azimuthal angles can be improved by accounting for the presence of the arms along the human sides. The human absorption at lower frequencies, i.e., few hundreds of $\mathrm{MHz}$, is not included is this study. This will be part of future research. 


\section{Acknowledgment}

This research has received funding from the Fund for Scientific Research - Flanders (FWO-V, Belgium) project G.0325.11N. Emmeric Tanghe is a Post-Doctoral Fellow of the FWO-V (Research Foundation - Flanders).

\section{Appendix A. Numerical determination of the body surface area (BSA) of heterogeneous phantoms}

The procedure to determine numerically the body surface area of the heterogeneous phantoms is presented here. Consider a certain phantom (Thelonious for instance) located in a rectangular parallelepiped limiting the simulation domain. The rectangular cuboid is discretized with a uniform grid size $h$. The discretization is performed with SEMCAD-X. After the discretization, each cube or voxel contains either a certain human tissue or the air surrounding the phantom indicated by an index. Fig. A1 shows the cross section of the discretized rectangular cuboid for a certain height $\mathrm{z}_{k}, \mathrm{k}=\{1, \ldots$, $\left.\mathrm{k}_{\max }\right\}$, where $\mathrm{k}_{\max }$ is the number of voxels along the $\mathrm{z}$-axis. $\mathrm{z}_{k}$ is the discrete height defining a cross section plane. Since Fig. A1 is in 2D, we use now the term pixel instead of voxel. In Fig. A1, a white pixel designates the air surrounding the phantom while

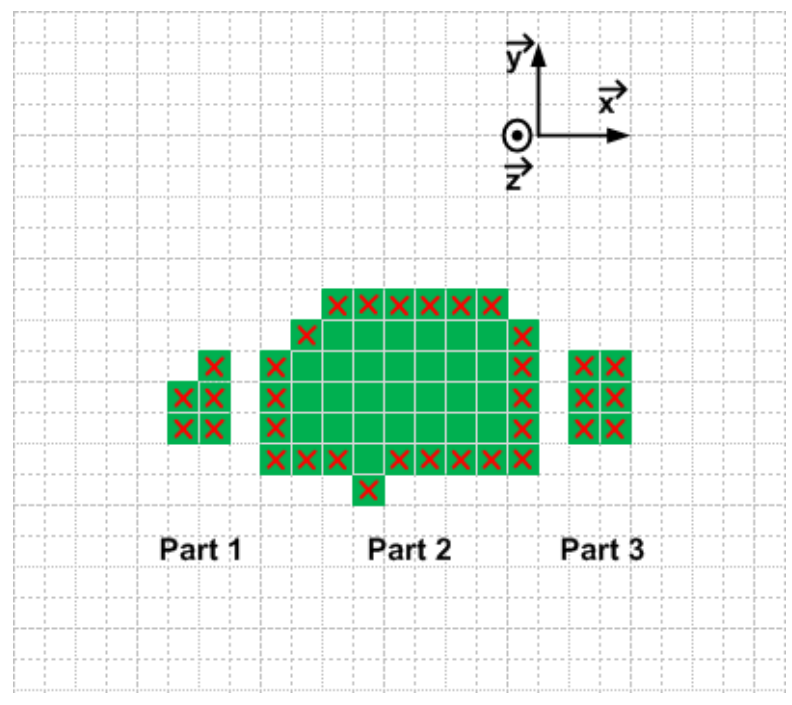

Figure A1. Cross section of the rectangular cuboid at a certain height $z_{k}$. The height is chosen such that the cross plan meets the human body.

a green pixel indicates a certain human tissue. Part 1, Part2, and Part 3 in Fig. A1 are the discretized right arm, trunk, and left arm, respectively. This means that the considered cross section of Fig. A1 is somewhere between Thelonious's hip and chest. For a given height $\mathrm{z}_{k}$, the BSA is the sum of the surfaces of the pixels adjacent to the air. Those pixels are indicated with a red cross in Fig. A1. Therefore, the BSA is obtained 
by summing all the surfaces of the adjacent pixels to the air, and for all $z_{k}$.

$$
\begin{aligned}
B S A & =\sum_{z_{k}} N_{p}^{a}\left(z_{k}\right) \times h^{2} \\
& =h^{2} \times \sum_{z_{k}} N_{p}^{a}
\end{aligned}
$$

where $h, \mathrm{~N}_{p}^{a}\left(\mathrm{z}_{k}\right)$ are the grid size and the number of pixels adjacent to the air in the cross plane $\mathrm{z}_{k}$, respectively. Note that the grid size in Fig. A1 is $25 \mathrm{~mm}$. We set such coarse grid size for visualization purposes. However, the grid size in the implementation was set to $5 \mathrm{~mm}$. Note that the numerical BSA slightly increases as the grid size decreases. For instance, a grid size of $2 \mathrm{~mm}$ results in an increase of the BSA of only $1 \%$ for Thelonious, while the simulation time is 25 times higher. The simulation time was about $3 \mathrm{mn}$ for a grid size of $5 \mathrm{~mm}$ while it was about $80 \mathrm{mn}$ for a grid size of $2 \mathrm{~mm}$.

\section{Appendix B. Numerical determination of the total projected body surface area $\left(\mathrm{BSA}_{p r}^{T}\right)$ of heterogeneous phantoms}

Recall that the $\mathrm{BSA}_{p r}^{T}$ is the sum of the projected human body surface from all the azimuthal angles, and is expressed as follows, see (3).

$$
B S A_{p r}^{T}=\int_{0}^{2 \pi} B S A_{p r}(\varphi) d \varphi
$$

where $\varphi$ is the azimuthal angle, and $\operatorname{BSA}_{p r}(\varphi)$ is the projected surface onto a plane along the azimuth $\varphi$.

The discretized rectangular cuboid described in Appendix A is used again to determine the $\mathrm{BSA}_{p r}^{T}$, and let us consider the same height $\mathrm{z}_{k}$ of Fig. A1. The human tissues are circumscribed in a rectangle ABCD in a $(0, X, Y)$ coordinate system, as shown in Fig. B1. Let us now define the coordinates of the points in Fig. B1.

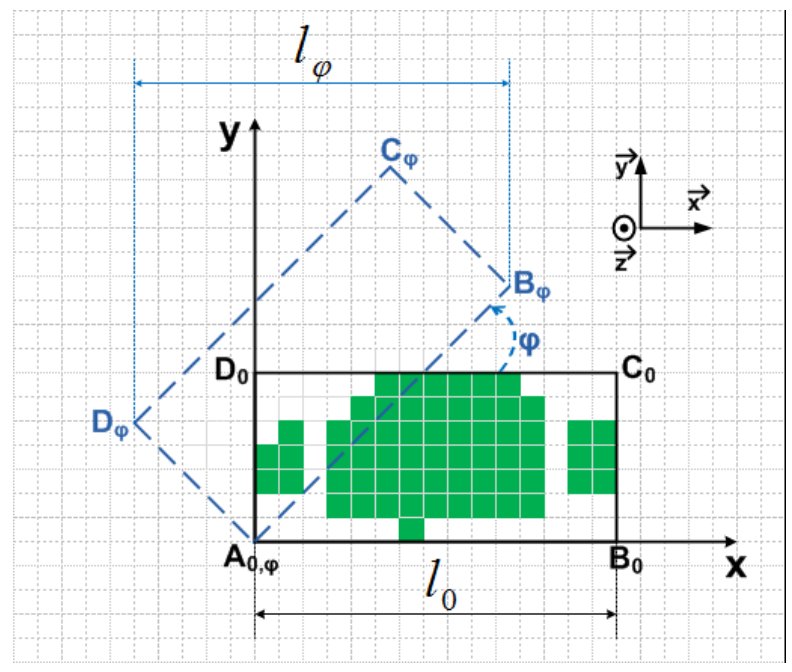

Figure B1. Human tissues circumscribed in a rectangle and its rotation. 
$\mathrm{A}_{0, \varphi}=\mathrm{A}_{0}=\mathrm{A}_{\varphi}=\left(\begin{array}{ll}0 & 0\end{array}\right)^{\prime} ; \mathrm{B}_{0}=\left(\begin{array}{ll}\mathrm{x}_{0} & 0\end{array}\right)^{\prime} ; \mathrm{C}_{0}=\left(\begin{array}{ll}\mathrm{x}_{0} & \mathrm{y}_{0}\end{array}\right)^{\prime} ; \mathrm{D}_{0}=\left(\begin{array}{ll}0 & \mathrm{y}_{0}\end{array}\right)^{\prime}$, where ${ }^{\prime}$ is the transpose notation. $\mathrm{x}_{0}$ and $\mathrm{y}_{0}$ are the maximum length of the phantom along the $\mathrm{X}$ and $\mathrm{Y}$ axis, respectively. In other words, $\mathrm{x}_{0}$ and $\mathrm{y}_{0}$ are the length and width of the phantom in the cross plane $z_{k}$. One can obtain their values since the grid size $h$ is known and the number of voxels (precisely pixels since the figure is in 2D) at each line can be easily determined. In Fig. B1, $\mathrm{x}_{0}=\mathrm{N}_{p}^{X} \times h$, and $\mathrm{y}_{0}=\mathrm{N}_{p}^{Y} \times h$ where $\mathrm{N}_{p}^{X}$ and $\mathrm{N}_{p}^{Y}$ are the maximum number of pixels along the $\mathrm{X}$ axis and the $\mathrm{Y}$ axis, respectively. $\mathrm{N}_{p}^{X}$ (resp. $\left.\mathrm{N}_{p}^{Y}\right)=13$ (resp. =7) in Fig. B1. The goal is first to determine the length $1_{\varphi}$ (see Fig. B1) of the projected rectangle $\mathrm{A}_{\varphi} \mathrm{B}_{\varphi} \mathrm{C}_{\varphi} \mathrm{D}_{\varphi}$ onto the $\mathrm{X}$ axis. The projected surface at azimuth $\varphi$ and cross plane $\mathrm{z}_{k}$ is defined by $\mathrm{l}_{\varphi}$ and the grid size $h$.

At the azimuth angle $\varphi$, the phantom is rotated with respect to $\varphi$. The coordinates of the rotation (along $\varphi$ ) of a certain point $\mathrm{M} \in\{\mathrm{A}, \mathrm{B}, \mathrm{C}, \mathrm{D}\}$ are as follows: $\mathrm{M}_{\varphi}=\mathrm{R}(\varphi) \cdot \mathrm{M}_{0}$, where $\mathrm{R}(\varphi)$ is the rotation matrix defined as:

$R(\varphi)=\left(\begin{array}{c}\cos (\varphi)-\sin (\varphi) \\ \sin (\varphi) \cos (\varphi)\end{array}\right)$.

Let $\mathrm{M}(\varphi)$ be defined as $\mathrm{M}(\varphi)=\left(\mathrm{x}_{M(\varphi)} \mathrm{y}_{M(\varphi)}\right)^{\prime}$. The length $\mathrm{l}_{\varphi}$ at a certain discrete height $\mathrm{z}_{k}$ is given by:

$$
l_{\varphi}^{z_{k}}=\max \left(x_{A(\varphi)}, x_{B(\varphi)}, x_{C(\varphi)}, x_{D(\varphi)}\right)-\min \left(x_{A(\varphi)}, x_{B(\varphi)}, x_{C(\varphi)}, x_{D(\varphi)}\right)
$$

where $\max ($.$) and \min ($.$) are the maximum and minimum value of the set between the$ brackets, respectively. Once $l_{\varphi}$ can be determined for a certain $\mathrm{z}_{k}$, the $\mathrm{BSA}_{p r}^{T}$ is given as:

$$
\begin{aligned}
B S A_{p r}^{T} & =\sum_{z_{k}} \sum_{\varphi} l_{\varphi}^{z_{k}} \times h \\
& =h \times \sum_{z_{k}} \sum_{\varphi} l_{\varphi}^{z_{k}}
\end{aligned}
$$




\section{References}

IEEE Standards Coordinating Committee 34 Recommended Practice for Determining the Peak Spatial-Average Specific Absorption Rate (SAR) in the Human Head from Wireless Communications Devices: Measurement Techniques 2003 Institute of Electrical and Electronics Engineers, Inc.

Foundation for Research on Information Technologies in Society (IT'IS) http://www.itis.ethz.ch/itis-for-health/virtual-population/human-models/ (accessed on April 4, 2013)

NIST: National Institute of Standards and Technology http://dlmf.nist.gov/19.33 (accessed on April 4, 2013)

Andersen J B, Rappaport T S and Yoshida S 1995 Propagation measurements and models for wireless communications channels IEEE Commun. Mag. 42-49

Andersen J B, Nielsen J Ø, Pedersen G F, Bauch G and Herdin M 2007 Room Electromagnetics IEEE Trans. Antennas Mag. 49 27-33

Andersen J B, Chee K L, Jacob M, Pedersen G F and Kürner T 2012 Reverberation and Absorption in an Aircraft Cabin with the Impact of Passengers IEEE Trans. Antennas Propag. 60 2472-80

Bakker J F, Paulides M M, Christ A, Kuster N and van Rhoon G C 2010 Assessment of induced $\mathrm{SAR}$ in children exposed to electromagnetic plane waves between $10 \mathrm{MHz}$ and $5.6 \mathrm{GHz}$ Phys. Med. Biol. 55 3115-30. Erratum in Phys. Med. Biol. 2011 May 7 56(9) 2883

Bamba A, Joseph W, Andersen J B, Tanghe E, Vermeeren G, Plets D, Nielsen J Ø and Martens L 2012 Experimental Assessment of Specific Absorption Rate Using Room Electromagnetics IEEE Trans. Electromagn. Compat. 54 747-57

Bamba A, Joseph W, Vermereen G, Tanghe E, Gaillot D P, Andersen J B, Nielsen J Ø, Lienard M and Martens L 2013 Validation of Experimental Whole-body SAR Assessment Method in a Complex Environment Bioelectromagnetics 34 122-32

Bamba A, Gaillot D, Tanghe E, Joseph W, Lienard M and Martens L 2013 Assessing Human Whole-Body Specific Absorption Rate For diffuse Exposure From Reverberation Chamber Measurements. submitted to IEEE Trans. Electromagn. Compat. (accepted under revision conditions).

Bolte J F, Van der Zande G, Kamer J 2011 Calibration and Uncertainties in Personal Exposure Measurements of RadioFrequency Electromagnetic Fields Bioelectromagnetics 32(8) 652-63

Chizhik D and Ling J 2008 Propagation Over Clutter: Physical Stochastic Model IEEE Trans. Antennas Propag. 56(4) 1071-77

Conil E, Hadjem A, Gati A, Wong M F and Wiart J 2011 Influence of Plane-Wave Incidence Angle on Whole-Body and Local Exposure at $2100 \mathrm{MHz}$ IEEE Trans. Electromagn. Compat. 53 48-52

Conil E, Hadjem A, Lacroux F, Wong M F and Wiart J 2008 Variability analysis of SAR from $20 \mathrm{MHz}$ to $2.4 \mathrm{GHz}$ for different adult and child models using finite-difference time-domain Phys. Med. Biol. 53 1511-25

Dimbylow P J 2002 Fine resolution calculations of SAR in the human body for frequencies up to $3 \mathrm{GHz}$ Phys. Med. Biol. 47 2835-46

Du Bois D and Du Bois E F 1916 A formula to estimate the appropriate surface area if height and weight be known Arch. Intern. Med. 17 863-71

Durney C H, Massoudi H and Iskander M F 1986 Radiofrequency Radiation Dosimetry Handbook Brooks Air Force Base-USAFSAM-TR-85-73

Fujimoto S, Watanabe T, Sakamoto A, Yukawa K and Morimoto K 1968 Studies on the physical surface area of Japanese. 18. Calculation formulas in three stages over all ages Nippon Eiseigaku Zasshi 5 443-50

Gehan E A and George S L 1970 Estimation of human body surface area from height and weight Cancer Chemoth. Rep. 54 225-35

Hill A D 1995 Spatial Correlation Function for Field in a Reverberation Chamber. IEEE Trans. 
Electromagn. Compat. 37 138-45

Hirata A, Fujiwara O, Nagaoka T and Watanabe S 2010 Estimation of whole-body average SAR in humans models due to plane wave exposure at resonance frequency IEEE Trans. Electromagn. Compat. 52 41-48

Hirata A, Ito N and Fujiwara O 2009 Influence of electromagnetic polarization on the whole-body averaged SAR in children for plane-waves exposures Phys. Med. Biol. 54 N59-N65

Hirata A, Kodera S, Wang J and Fujiwara O 2007 Dominant factors influencing whole-body average SAR due to far-field exposure in whole-body resonance frequency and $\mathrm{GHz}$ regions Bioelectromagnetics $\mathbf{2 8}$ 484-87

International Commission on Non-Ionizing Radiation Protection 1998 Guidelines for limiting exposure to time-varying electric, magnetic, and electromagnetic fields (up to $300 \mathrm{GHz}$ ) Health Phys 74 494-522

Joseph W, Vermeeren G, Verloock L, Heredia M M, Martens L 2008 Characterization of Personal RF Electromagnetic Field Exposure and Actual Absorption for the General Public Health Phys 95(3) 317-30

Joseph W, Vermeeren G, Verloock L, Martens L 2010 Estimation of Whole-Body SAR From Electromagnetic Fields Using Personal Exposure Meters Bioelectromagnetics 31 286-95

Kühn S, Jennings W, Christ A and Kuster N 2009 Assessment of induced radio-frequency electromagnetic fields in various anatomical human body models Phys. Med. Biol. 54 875-90

Kuttruff H 2000 Room Acoustics Spon Press, London, UK, 4th Edition

Livingston E H and Lee S 2001 Body surface area prediction in normal weight and obese patients Am. J. Physiol. Endoc. M. 281 E586-E591

Primiani V M, Moglie F 2012 Numerical Simulation of Reverberation Chamber Parameters Affecting the Received Power Statistics IEEE Trans. Electromagn. Compat. 54 522-32

Poutanen J, Salmi J, Haneda K, Kolmonen V M and Vainikainen P 2011 Angular and Shadowing Characteristics of Dense Multipath Components in Indoor Radio Channels IEEE Trans. Antennas Propag. 59 245-53

Richter A, Thoma R S 2003 Parametric Modeling and Estimation of Distributed Diffuse Scattering Components of Radio Channels COST273 TD(03)198 http://www.lx.it.pt/cost273/

Richter A 2005 Estimation of radio channel parameters: Models and algorithms $(\mathrm{PhD}$ thesis ) Faculty of Electrical Engineering and Information Technology at the Ilmenau University of Technology, Ilmenau, Germany

Röösli M, Frei P, Mohler E, Braun-Fahrländer C, Burgi A, Fröhlich J, Neubauer G, Theis G, Egger M 2008 Statistical Analysis of Personal Radiofrequency Electromagnetic Field Measurements with Nondetects Bioelectromagnetics 29(6) 471-78

Saleh A A M and Valenzuela R A 1987 A Statistical Model for Indoor Multipath Propagation IEEE J. Select. Areas Commun. JSAC-5 128-37

Smith F W, Merewether D E and Fisher R 1980 On implementing a numeric Huygen's source scheme in a finite difference program to illustrate scattering bodies IEEE Trans. Nucl. Sci. $\mathbf{2 7}$ 1829-33

Taflove A and Hagness S 2005 Computational electrodynamics: The finite-difference time-domain method Norwood, MA, 3rd edition

Tanghe E, Joseph W, Lienard M, Nasr A, Stefanut P, Martens L and Degauque P 2011 Statistical Analysis of Multipath Clustering in Indoor Office Environments EURASIP Journal on Wireless Communications and Networking 2011. http://jwcn.eurasipjournals.com/content/2011/1/26313416 pages

Thielens A, De Clercq H, Agneessens S, Lecoutere J, Verloock L, Declercq F, Vermeeren G, Tanghe E, Rogier H, Puers R, Martens L, Joseph W 2013 Distributed On Person Exposimeters for Radio Frequency Exposure Assessment in Real Environments Bioelectromagnetics 34(7) 563-67

Ullmo D and Baranger H U 1999 Wireless Propagation in Buildings: A Statistical Scattering Approach IEEE Trans. Veh. Technol. 48(3) 947-55 
Uusitupa T, Laakso I, Ilvonen S and Nikoskinen K 2010 SAR variation study from $300 \mathrm{MHz}$ to $5000 \mathrm{MHz}$ for 15 voxels models including different postures Phys. Med. Biol. 55 1157-76

Vermeeren G, Joseph W and Martens L 2008 Whole-body SAR in spheroidal adult and child phantoms in a realistic exposure environment IET Electron. Lett. 44 790-91

Vermeeren G, Joseph W, Olivier C and Martens L 2008 Statistical multipath exposure of a human in a realistic electromagnetic environment Health Phys 94 345-54

Wang J, Fujiwara O, Kodera S and Watanabe S 2006 FDTD calculation of whole-body average $\mathrm{SAR}$ in adult and child models for frequencies from $30 \mathrm{MHz}$ to $3 \mathrm{GHz}$ Phys. Med. Biol. 51 4119-27 